\title{
Achieving the CSHP's Vision for Hospital Pharmacy in Canada
}

Patricia Macgregor

$\mathrm{T}^{\mathrm{h}}$ he profession of pharmacy is in the midst of significant change with respect to scope of practice. Provincial governments, in collaboration with pharmacy regulatory authorities, are in the process of legislating expanded scopes of practice for pharmacists and regulating the practice of pharmacy technicians. Both pharmacists and technicians have opportunities to enhance their roles within the health care team.

We asked for and have received an expanded scope of practice, but are we adopting it? How many pharmacists will become certified to administer injections? How many will modify prescriptions? What percentage of pharmacy assistants will submit to regulation and practice to full scope? Yes, there are many questions. The provincial ministries of health have placed their trust in us, but are we ready to rise to the criteria implied by their confidence? We can certainly learn from our interprofessional colleagues who have travelled this path before us. For example, nurses and respiratory therapists readily adopted advanced scopes of practice (including activities such as prescribing) in hospitals, family health teams, and home care. Pharmacists' adoption of advanced scope of practice activities provides an opportunity to enhance the visibility of our profession to the public, the health care team, ministries of health, and other key stakeholders.

In addition to creating new roles, these changes also create new demands and pressures. CSHP is helping its members to deal with these new demands and to gain recognition for the value of our work in patient care. In particular, the CSHP 2015 initiative outlines excellent, measurable activities that represent opportunities not only to enhance our practice but also to communicate the impact of our work within our respective organizations and beyond. The key clinical performance indicators, which are currently being developed through a robust Delphi process and are to be unveiled at the Professional Practice Conference (PPC) in February 2013, will enable us to specifically promote the value of our clinical activities. The CSHP Advocacy Committee is diligently engaging key stakeholders to highlight the value of pharmacists in the circle of care.
CSHP is also responding to the changing needs of members for communication, participation, and learning by building and expanding digital communication channels. You can join CSHP on Facebook, participate in CSHP 2015 blogs and Twitter streams, and take advantage of the topical education webinars.

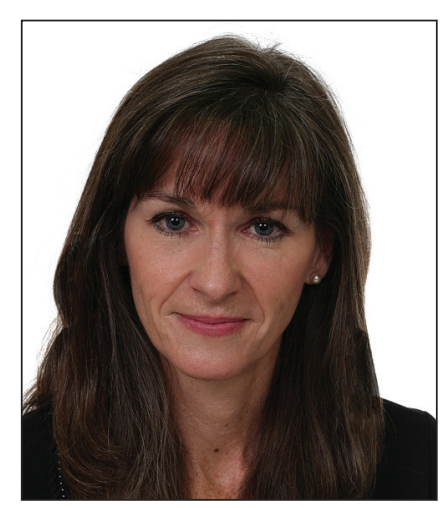

During the PPC, check out award-winning residency projects, virtual posters, and student video competition entries, and consider participating in next year's awards programs.

You can also connect with and learn from colleagues by joining one of CSHP's 23 Pharmacy Specialty Networks (PSNs), which cover diverse areas such as antimicrobial stewardship, drug utilization, emergency, home care, pediatrics, and transplantation. There is even a PSN specifically for pharmacists practising in small hospitals. Participating members rate PSNs as informative with regard to new practices and guidelines, ideas on how to better serve patients and share knowledge, connecting with peers across Canada, and more. What better way to connect efficiently and productively with colleagues with similar interests and expertise that they are willing to share? Check www.cshp.ca for information.

Pharmacists are creative, innovative, and dedicated professionals, keen to collaborate to advance patient care. I encourage you to take the initiative: participate, lead, be engaged, and promote our profession.

Patricia Macgregor, BSc, RPh, MRPharmS, MHSC, CHE, is President Elect and Vision Liaison for the Canadian Society of Hospital Pharmacists. 